International Journal of Engineering \&Technology, $7(1.8)(2018) 123-129$
International Journal of Engineering \& Technology
SPC
Website: www.sciencepubco.com/index.php/IJET
Research paper

\title{
Performance analysis of two stage adaptive FIR Filter Algorithms for PLI and BW artifact cancellation in ECG
}

\author{
B. Bhaskara Rao ${ }^{1 *}$, B. Prabhakara Rao ${ }^{2}$ \\ ${ }^{1}$ Research Scholar, ECE Dept., JNTUniversity:Kakinada,Kakinada 533003, India \\ ${ }^{2}$ Program Director,School of Nanotechnology,IST,Kakinada 533003, India \\ *Corresponding author E-mail: b.bhaskerrao.ece@gmail.com
}

\begin{abstract}
Electrocardiogram (ECG) is a measure of the electrical movement of the heart, and is obtained by surface electrodes at standardized locations on the patient's chest. During acquisition, various artifacts/noises such as power-line interference (PLI), baseline wander (BW), muscle artifacts (MA) and motion artifacts (EM) obscure the ECG. It is important that these artifacts are minimized for the clinicians to make better diagnosis on heart problems. This paper researches the creative idea of adaptive noise cancellation (ANC) using two stage form of adaptive filters. The concept of cascading and its algorithm for real-time application is simulated on MATLAB. The proposed algorithm utilizes two adaptive filters to estimate gradients accurately which results in good adaptation and performance. The objective of the present investigation is to provide solution in order to improve the performance of noise canceller in terms of filter parameters which are obtained with the help of adaptive algorithms. Different kinds of two stage ANC algorithms are used to eliminate artifacts in ECG by considering the noises such as power line interference and baseline wander. The simulation results show that the performance of the two stage ANC is superior to the conventional single stage ANC system in terms of higher signal-to-noise ratio. Two stage adaptive algorithms are applied on real time ECG signals and compared their performance with the conventional single stage adaptive algorithms in terms of parameters Signal-to-Noise Ratio (SNR), Mean Square Error (MSE), Root Mean Square Error (RMSE) and Distortion.
\end{abstract}

Keywords: ECG, PLI, BW, MA, EM, ANC, MIT-BIH, SNR, MSE, RMSE, Distortion

\section{Introduction}

Signals play important role in the field of medical, electrical, electronic and communication engineering. The signals related to medical field are known as biomedical signals. Various biomedical signals like ECG, EEG and EMG are used for diagnosis as they contain lot of information. The biomedical signals are classified with regard to their source and application in terms of the signal characteristics and can be considered to be continuous or discrete. Continuous signals include temperature, pressure and chemical concentration, while electrical impulses generated by individual nerve cells can be considered as discrete signals[1,2]. ECG is an important biomedical instrument for the diagnosis of cardiovascular issue, that reported to the electrical activity of heart recorded by skin electrode. The morphology and the heart rate reflect the cardiac health of human heart beat and it is a non intrusive system that is measured on the surface of the human body, which is used in identification of heart diseases. Any disorder of pulse or beat, or an adjustment in the morphological pattern, is an indication of cardiac arrhythmia, which could be identified by analysis of recorded ECG waveform [2]. ECG signal represents an extremely important measure used by doctors, as it provides vital information about a patient's cardiac condition and general health. Generally the frequency band of the ECG signal is $0.05 \mathrm{~Hz}$ to 100 $\mathrm{Hz}$. The goal of any filter is to extract useful information from noisy data. A normally fixed filter is outlined ahead of time with the knowledge of the signal measurements and undesirable noise, however in the event that the noise statistics are not known from the earlier, or change after some time, the filter coefficients cannot be determined from in advance. In these situations, adaptive algorithms are needed in order to continuously update the filter coefficient $[3,4,5]$. Adaptive filtering finds application in noise cancellation called as adaptive noise cancellation (ANC) which involves in time-varying signals and systems $[6,7]$. ANC is an effective method for recovering a signal corrupted by additive noise and it is an important core area of the digital signal processing [3]. Figure 1 demonstrates the basic problem and the adaptive noise cancelling solution. A signal $s(n)$ is transmitted over a channel to a sensor that also receives a noise $\mathrm{n} 1(\mathrm{n})$ uncorrelated with the signal $[8,9]$. The primary input to the canceller is combination of signal and noise i.e. $s(n)+n 1(n)$. A second sensor receives a noise $n 2(n)$ uncorrelated with the signal but correlated with the noise $\mathrm{n} 1(\mathrm{n})$ that provides the reference input to the canceller which is filtered to produce an output $y(n)$ that is close a replica of $n 1(n)$. The output of the adaptive filter is subtracted from the primary input to produce the adaptive filter error as shown in equation (1) [10]. $e(n)=d(n)-y(n)$

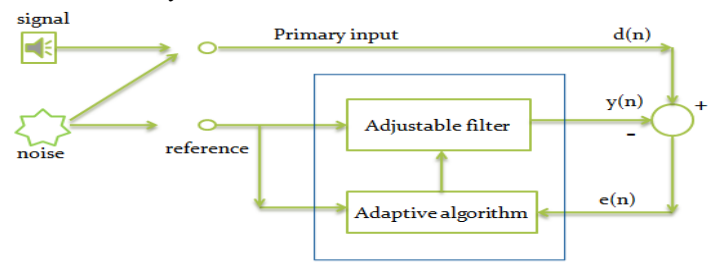

Fig. 1: Adaptive Noise Canceller 
Adaptive filtering algorithms [5], which constitute the modification system for the filter coefficients, are in certainty firmly identified with the established advancement procedures in spite of the fact that, in the last mentioned, every one of the computations are completed in a non-linear manner. An adaptive filter, due to its real-time self-adjusting characteristic, is expected to track the optimum [3] behavior of a slowly varying environment. Figure 1 demonstrates a block diagram in which a sample from a digital reference input $x(n)$ is fed into a device, called an adaptive filter, that computes a corresponding output sample $y(n)$ at time $n$. As the time index $\mathrm{n}$ is incremented, it is estimated that the output of the adaptive filter becomes a better and better match to the desired response signal through this adaptation process, such that the magnitude of e(n) decreases over time. An adaptive algorithm is a set of recursive equations used to adjust the weight vector $\mathrm{w}(\mathrm{n})$ automatically to minimize the error signal e(n) such that the weight vector converges iterative to the optimum solution i.e. the minimum MSE[3]. The least-mean-square (LMS) algorithm is the most widely used adaptive algorithms due to its simplicity and robustness. The factors that determine the performance of an algorithm are rate of convergence, mis adjustment, numerical robustness, computational requirements and stability are clearly stated in $[3,4,5]$.

Conventional filters,for example, the finite impulse response (FIR) filters [11], infinite impulse response (IIR) filters [12], filter banks [13], polynomial filter [14] and wiener filter are proposed in the literature to limit artifacts. Different methodologies for ECG denoising include adaptive filters, namely the least mean square (LMS) and their variants such as normalized least mean square (NLMS), variable step size least mean square (VSLMS) and variable step size normalized least mean square (VSNLMS) [15, 16 $17,18,19,20,21,22,23,24,25,26,27]$ etc... A few researchers like wise recommended adaptive kalman filter and extended kalman filter. [28]. Promising performances are obtained by nonlinear techniques, for example, the bayesian filtering and nonlinear projective filtering $[29,30]$.

Strategies for decomposition of signals into subcomponents for noise lessening have turned out to be prominent and were proposed to eliminate noise from ECG signals that incorporate independent component analysis(ICA) $[31,32]$, singular value decomposition (SVD), empirical mode decomposing (EMD), and ensemble EMD (EEMD) [33, 34, 35]. Soft, hard and adaptive thresholding techniques are also proposed on EMD and EEMD plans $[36,37]$. Wavelet transformation (WT) has been a powerful instrument for denoising signals in the frequency domain $[38,39]$ and is proposed for ECG denoising.

It has conventionally been used by applying soft or hard thresholds on the obtained discrete wavelet transform (DWT) coefficients[40, 41]. A combination of DWT with wiener filtering has been proposed by Kesler et al., [42, 43]. Recently, crossover plans have been proposed to enhance noise elimination execution. For instance, in [16], the EMD and EEMD strategies were utilized to provide the reference input to the block LMS adaptive filter. An adaptive filter approach for decreasing the noise of the ECG signal utilizing neural systems to limit artifacts on the ECG has been appeared to give preferred execution over traditional wavelet techniques [44, 45, 46, 47]. In this paper, the comprehensive analysis of two stage adaptive FIR filter schemes for denoising ECG signals is presented. The single stage adaptive FIR filter based noise canceller method is cascaded with another single stage adaptive FIR filter, namely the two stage adaptive noise canceller approach. The performance results of this method are compared to the conventional single stage adaptive filter methods by performing broad simulation contemplates on on real time noise corrupted ECG signals obtained from the standard data base[48].These two stage techniques enhance the capacity to adapt the time varying nature of ECG and limit the noises with a minimum signal distortion.

\section{Background}

In order to review the ECG of the patient in real-time, there is a possibility that the ECG signal might be contaminated by noise. The predominant artifacts present in the ECG includes: power-line interference (PLI), baseline wander (BW), muscle artifacts (MA) and motion artifacts (EM), mainly caused by patient breathing, movement, power line interference, bad electrodes and improper electrode site preparation. The low frequency segments of ECG signals are strongly influenced by this contamination which prompts a false diagnosis. The ECG is a moderately strong signal with an effectively identifiable waveform. Most types of interference that affect ECG signals are removed by band pass filters that may not give best outcome. The disturbances in ECG signal need to be reduced to improve the accuracy and reliability for better diagnosis [49]. Many methods are implemented to remove the noise from noisy ECG signal[50]. The basic method is to pass the signal through static filters such as high pass, low pass and notch filters. However, the disadvantage of the static filter is that filtering or preprocessing of an ECG signal need to be done by understanding the region of noise cut off frequencies present in the signal. The static filters have fixed filter coefficients and it is difficult to reduce the instrumentation noise due to the time varying behavior that is unknown. In order to overcome these limitations of static filters, different adaptive filtering methods $[51,52]$ are developed. Adaptive filtering uses variety of algorithms such as LMS algorithm, NLMS algorithm, VSLMS algorithm and VSNLMS algorithm etc. The performance of these adaptive filtering algorithms is dependent on their filter length $(\boldsymbol{N})$ and the convergence parameter $(\boldsymbol{\mu})$ commonly known as step size or learning rate parameter. The main drawback of the pure LMS algorithm is that it is sensitive to the scaling of its input $\boldsymbol{u}(\boldsymbol{n})$ that makes it very hard to choose a learning rate $\boldsymbol{\mu}$ that is constant throughout every iteration upon which the stability of the algorithm depends. The NLMS algorithm is a variant of the LMS algorithm that solves drawback of the LMS algorithm by normalizing power of the input. However NLMS algorithm [23] overcomes the drawback of constant step size parameter by varying at every iteration in accordance with every instant of the signal. The drawback of VSLMS algorithm is overcome by VSNLMS algorithm by calculating instantaneous input signal energy at every iteration [26].

\section{Proposed Methodology}

LMS algorithm[17, 21, 22] is most commonly used adaptive algorithm than RLS and kalman filter which uses a gradient vector to estimate a time-varying signal. The gradient is the del operator that is applied to find the estimate of a function which is the error with respect to the $\boldsymbol{n}^{\text {th }}$ coefficient at every instant of time. The concept of cascading adaptive FIR filters is presented in [19] with cascading using LMS adaptive filter.

In this method the input signal given to the first adaptive filter consists of original clean ECG signal s(n) and noise $\boldsymbol{n 1 ( n )}$ which is denoted as primary signal $\boldsymbol{d}(\boldsymbol{n})$ in Figure 2. The secondary noise or the reference noise signal $\boldsymbol{u}(\boldsymbol{n})$ given to adaptive filter is noise signal $\boldsymbol{n}_{2}(\boldsymbol{n})$. The output of the adaptive filter $\boldsymbol{y}(\boldsymbol{n})$, subtracted from the primary signal $\boldsymbol{d}(\boldsymbol{n})$ gives the desired signal or the error signal $\boldsymbol{e}(\boldsymbol{n})$ for second adaptive filter [19]. 


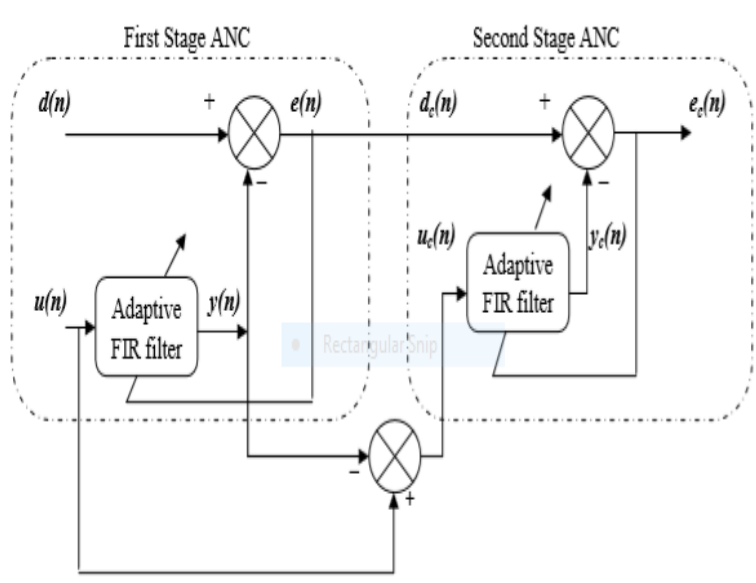

Fig. 2: Proposed Two Stage Adaptive Noise Canceller for ECG denoising

\subsection{Analysis of single stage adaptive algorithms}

The two stage adaptive noise canceller shown in Figure. 2 consists of first adaptive filter whose parameters $d(n)$ is the primary signal, $\boldsymbol{e}(\boldsymbol{n})$ is the desired signal or error signal, $\boldsymbol{y}(\boldsymbol{n})$ is the output of an adaptive FIR filter, $\boldsymbol{\mu}$ is the step size parameter, $\boldsymbol{w}(\boldsymbol{n})$ is the filter weight vector, $\boldsymbol{u ( n )}$ is the reference input signal vector, and $\boldsymbol{N}$ is the filter length used as parameters of first LMS filter. Here $\boldsymbol{u}(\boldsymbol{n})$ is the input vector of time delayed input values,

$u(n)=[u(n), u(n-1), u(n-2), \ldots \ldots \ldots \ldots . . . . u(n-N+1)]^{T}$, where the superscript $\mathrm{T}$ denotes the transpose operation of the matrix. The vector $w(n)=\left[w_{0}(n), w_{1}(n), w_{2}(n), \ldots . . w_{N-1}(n)\right]^{T}$ represents the coefficients of the adaptive FIR filter tap weight vector at time $\boldsymbol{n}$. Selection of a suitable value for step size parameter $\boldsymbol{\mu}$ is imperative to the performance of the adaptive algorithms, i.e. if $\boldsymbol{\mu}$ is too small the time taken by the adaptive filter to converge on the optimal solution will be too long; and if $\boldsymbol{\mu}$ is too large then the adaptive filter becomes unstable and its output diverges[3, 4, 5]. The single stage adaptive algorithms require three distinct computational steps in each iteration as follows.

1. The output of the first stage adaptive FIR filter, $\boldsymbol{y}(\boldsymbol{n})$ is calculated using equation

$y(n)=\sum_{j=0}^{N-1} w(n) x(n-i)=w^{T}(n) x(n)$

2. The value of the error or cost function i.e. the difference between primary signal and estimated adaptive filtered output signal is calculated using equation (3)

$e(n)=d(n)-y(n)$

3. The tap weight vectors or filter coefficients of the first stage adaptive FIR filter are updated in a sample by sample manner for the next iteration by using equations (4)-(8) for different adaptive algorithms.

\section{i) Single Stage LMS (SSLMS) Algorithm}

$$
w(n+1)=w(n)+\mu e(n) u(n)
$$

To guarantee the stability of the algorithm, the step size is chosen in the range

$$
0<\mu<\frac{2}{N * P_{u}}
$$

Here the parameter $\boldsymbol{P}_{\boldsymbol{u}}$ is average power of the input signal $\boldsymbol{u}(\boldsymbol{n})$ and it is calculated as

$$
\boldsymbol{P}_{\boldsymbol{u}}=u^{T}(n) u(n)
$$

\section{ii) Single Stage NLMS (SSNLMS) Algorithm}

$\mathrm{w}(n+1)=\mathrm{w}(n)+\mu(n) e(n) \mathrm{u}(n)$

Here the time varying step size parameter $\boldsymbol{\mu}(\boldsymbol{n})$ value is calculated as

$\mu(n)=\frac{\mu}{\left(u^{T}(n) u(n)\right)}$

\subsection{Analysis of Two Stage Adaptive algorithms}

The two stage structure of ANC as shown in Figure. 2, consist of input signal $\boldsymbol{u}_{\boldsymbol{c}}(\boldsymbol{n})$ that is cascaded reference input signal vector and cascaded primary signal $\boldsymbol{d}_{\boldsymbol{c}}(\boldsymbol{n})$ [19]. The two stage adaptive algorithms require five distinct steps in each iteration as follows.

1. Compute the cascaded input signal vector $\boldsymbol{u}_{\boldsymbol{c}}(\boldsymbol{n})$ using equa$\operatorname{tion}(9)$

$u_{c}(n)=u(n)-y(n)$

2. The output of the second stage adaptive FIR filter, $\boldsymbol{y}_{\boldsymbol{c}}(\boldsymbol{n})$ is calculated using equation(10)

$y_{C}(n)=\sum_{j=0}^{N-1} w_{C}(n) u_{C}(n-i)=w_{C}^{T}(n) u_{c}(n)$

Here $\boldsymbol{u}_{\boldsymbol{c}}(\boldsymbol{n})$ and $\boldsymbol{w}_{\boldsymbol{c}}(\boldsymbol{n})$ are the input vector of time delayed cascaded reference input signal values, the tap weights of the second stage adaptive FIR filter

3. Assign first stage error signal to second stage as a cascaded primary signal $\boldsymbol{d}_{\boldsymbol{c}}(\boldsymbol{n})$

$d_{c}(n)=e(n)$

4. The value of the cascaded estimation error signal is calculated using equation (12)

$e_{c}(n)=d_{c}(n)-y_{c}(n)$

5. The tap weight vectors of the second stage adaptive FIR filter are updated in preparation for the next iteration by equations (13)(18) for different adaptive algorithms.

\section{i) Two Stage LMS (TSLMS) Algorithm}

$w_{c}(n+1)=w_{c}(n)+\mu_{c} u_{c}(n)$

Where $\mu_{c}$ is step size parameter.

ii) Two Stage NLMS (TSNLMS) Algorithm

$w_{c}(n+1)=w_{c}(n)+\mu_{c}(n) e_{c}(n) u_{c}(n)$

Here the time varying step size parameter $\boldsymbol{\mu}_{\boldsymbol{c}}(\boldsymbol{n})$ value is calculated as

$\mu_{c}(n)=\frac{1}{\left(u^{T}(n) u(n)\right)}$

Equation (16) is used to estimate the remaining error presented in the system as

$e_{c}(n)=d_{c}(n)-w^{T}(n) u(n)$

Final noise presented in the system is

$e(n)=d(n)-y(n)=s(n)+n_{1}(n)-n_{2}(n)=s(n)+n_{3}(n)$

Here $n_{3}(\boldsymbol{n})$ is the remaining artifact/noise presented in the error signal [19].

$n_{3}(n)=n_{1}(n)-n_{2}(n)$

\subsection{Performance Parameters of Two Stage ANC}

In the two stage ANC algorithm, the error signal $\boldsymbol{e}(\boldsymbol{n})$ of first adaptive filter is used as a primary signal $\boldsymbol{d}_{\boldsymbol{c}}(\boldsymbol{n})$ of second adaptive filter, the output $\boldsymbol{y}(\boldsymbol{n})$ of first adaptive filter subtracted from the reference 
noise signal $\boldsymbol{u}(\boldsymbol{n})$ is $\boldsymbol{u}_{\boldsymbol{c}}(\boldsymbol{n})$ serves as the reference noise signal for second adaptive filter. Therefore, the two stage adaptive algorithms are generations and simulations are performed by MATLAB for non-stationary environment. Desired signal $\boldsymbol{d}(\boldsymbol{n})$ is a noisy signal including clean ECG signal and the reference signal $\boldsymbol{u}(\boldsymbol{n})$ is the predominant artifacts(noise) present in the ECG which may be caused due to PLI,BW,MA and EM. The clean ECG signal can be extracted from primary signal. The performances of simulation of two stage ANC and single stage ANC are compared quantitatively by parameters Mean Square Error (MSE), Root Mean Square Error (RMSE), Signal-to-Noise Ratio (SNR) and Distortion $[3,4,5]$ summarized in Table 1 .

Table 1: The two stage ANC system quantitative

PARAMETERS

MSE

RMSE

SNR(Before Filtering)

SNR(After Filtering)

DISTORTION

\subsection{Computational Complexity of Two Stage ANC}

From the analysis of single stage and two stage adaptive algorithms of FIR systems can be viewed as a computational procedure to determine the output sequence from the input sequence. The basic elements used to realize the algorithms are constant multiplier, unit delay delay element and adder.The computational complexity figures required to compute all the versions of LMS, as proposed above are summarized in Table 2 .

Table 2: Computational Complexity

\begin{tabular}{lcc}
\hline \multicolumn{1}{c}{ ALGORITHMS } & $\begin{array}{c}\text { MULTIPLIERS } \\
\text { REQUIRED }\end{array}$ & $\begin{array}{c}\text { ADDERS } \\
\text { REQUIRED }\end{array}$ \\
\hline SSLMS & $2 \mathrm{~N}+1$ & $2 \mathrm{~N}$ \\
TSLMS & $4 \mathrm{~N}+1$ & $4 \mathrm{~N}+1$ \\
SSNLMS & $3 \mathrm{~N}+1$ & $2 \mathrm{~N}$ \\
TSNLMS & $6 \mathrm{~N}+1$ & $4 \mathrm{~N}+1$ \\
\hline
\end{tabular}

\section{Simulation Results and Discussion}

The benchmark MIT-BIH arrhythmia database [48] was used to test the performance of various adaptive algorithms for ECG denoising and it consists of 48 half hour excerpts of two channel ambulatory ECG recordings, which were obtained from 47 subjects, including 25 men aged 32-89 years, and women aged 23-89 years. The recordings were digitized at 360 samples per second per channel with 11-bit resolution over a $10 \mathrm{mV}$ range. The simulations were done by collecting 3600 samples of ECG recordings. In this simulation the initial filter weight vector for both the filters is $\mathrm{N}+1$ filter coefficients that are designed from an $\mathrm{N}$ th order low pass FIR digital filter with length same as the length of ECG record using kaiser window function with filter cut-off frequency 100 $\mathrm{Hz}$, sampling frequency $360 \mathrm{~Hz}$ and beta is 3 . A data set of five ECG records: data100, data105, data108, data203 and data228 are considered to ensure the consistency of the results. The reference signal n2(n) shown in Figure 1. is taken from noise generator. A synthetic PLI with $1 \mathrm{mv}$ amplitude is simulated for PLI cancellation where no harmonics are synthesized. In order to test the filtering capability in non-stationary environment real BW, MA and EM noises with 3600 samples are considered. These are taken from MIT-BIH normal sinus rhythm database (NSTDB) [53] which consists of 12 half-hour ECG recordings and 3 half-hour recordings of noise typical ambulatory ECG recordings. The noise recordings that occur due to physically active volunteers, standard ECG recorders, leads, and electrodes that were placed on the limbs in positions of the subjects' ECGs that contain predominantly baseline wander (in record 'bw'), muscle (EMG) artifact (in record 'ma'), and electrode motion artifact (in record 'em').

\subsection{Power-line Interference (PLI) Cancellation}

In this experiment 3600 samples of ECG signal which are corrupted with synthetic PLI having frequency of $60 \mathrm{~Hz}$, sampled at $200 \mathrm{~Hz}$ is applied as primary input to the adaptive filter shown in Figure 1. The Figures 3-4 depict the PLI cancellation by considering the number of samples and amplitude on $\mathrm{x}$-axis and $\mathrm{y}$-axis respectively. The performance of the adaptive filter algorithms are assessed by SNR, MSE, RMSE and Distortion Values for entire dataset. The two stage adaptive algorithms achieve SNR, MSE, RMSE improvement over the conventional single stage adaptive algorithms. Tables 3-6 shows improvement for the entire data set in terms of SNR, Mean Square Error (MSE), Root Mean Square Error (RMSE) and Distortion. The simulation results state that two stage adaptive algorithms have greater efficiency than single stage adaptive algorithms. It is clear from Table 3 that two stage adaptive algorithms outperform single stage adaptive algorithms in estimating the ECG noises. These results for data105 are shown in Figure $3 \& 4$.

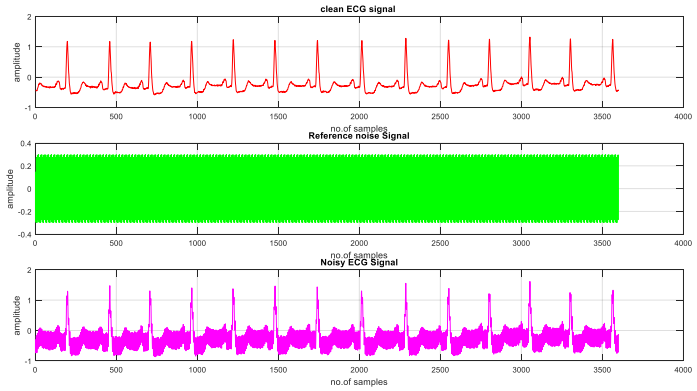

Fig. 3: Simulated model 1 filtering results of PLI Cancelation (a) Clean ECG Signal(red) for MIT-BIH Record Number 105 (b) Reference $60 \mathrm{~Hz}$ Noise Signal (green) (c) MIT-BIH Record 105 with 60Hz Noise (magenta)
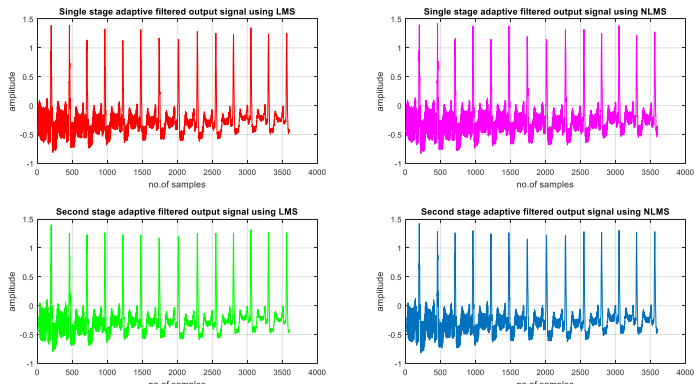

Fig. 4: Simulation results of PLI Cancelation for MIT-BIH Record Number 105 (a) Recovered (red) signal using SSLMS algorithm (b) Recovered (green) signal using TSLMS algorithm (c) Recovered(magenta) signal using SSNLMS algorithm (d) Recovered(blue) signal using TSNLMS algorithm

Table.3 SNR improvement for the dataset.

\begin{tabular}{|c|c|c|c|c|c|}
\hline Record No & SNRBF & SSLMS & TSLMS & SSNLMS & TSNLMS \\
\hline $\mathbf{1 0 0}$ & 4.6514 & 11.6926 & 13.7382 & 9.2262 & 11.6708 \\
\hline $\mathbf{1 0 5}$ & 5.1489 & 12.1569 & 14.2049 & 9.7039 & 12.1443 \\
\hline $\mathbf{1 0 8}$ & 4.4311 & 11.3987 & 13.4588 & 8.9616 & 11.4057 \\
\hline $\mathbf{2 0 3}$ & 7.4041 & 14.3968 & 16.4459 & 11.9644 & 14.3873 \\
\hline $\mathbf{2 2 8}$ & 3.3838 & 10.3824 & 12.4051 & 7.9334 & 10.3587 \\
\hline
\end{tabular}

Table. 3 shows the performance of SSLMS, TSLMS, SSNLMS, and TSNLMS algorithms in terms of SNR. The simulation results state that Two Stage Adaptive algorithms have greater efficiency than Single Stage 
Adaptive algorithms. It is clear from Table 3 that Two Stage Adaptive algorithms outperform Single Stage Adaptive algorithms in estimating the ECG noises.

Table.4 MSE improvement for the dataset.
\begin{tabular}{|c|c|c|c|c|}
\hline Record No & SSLMS & TSLMS & SSNLMS & TSNLMS \\
\hline $\mathbf{1 0 0}$ & 0.1406 & 0.1369 & 0.1473 & 0.1404 \\
\hline $\mathbf{1 0 5}$ & 0.1564 & 0.1529 & 0.1631 & 0.1563 \\
\hline $\mathbf{1 0 8}$ & 0.1339 & 0.1304 & 0.1406 & 0.1338 \\
\hline $\mathbf{2 0 3}$ & 0.2568 & 0.2531 & 0.2636 & 0.2566 \\
\hline $\mathbf{2 2 8}$ & 0.1072 & 0.1036 & 0.1139 & 0.107 \\
\hline
\end{tabular}

Table.5 RMSE improvement for the dataset.

\begin{tabular}{|c|c|c|c|c|}
\hline Record No & SSLMS & TSLMS & SSNLMS & TSNLMS \\
\hline $\mathbf{1 0 0}$ & 0.375 & 0.37 & 0.3838 & 0.3747 \\
\hline $\mathbf{1 0 5}$ & 0.3955 & 0.391 & 0.4039 & 0.3954 \\
\hline $\mathbf{1 0 8}$ & 0.366 & 0.361 & 0.375 & 0.3658 \\
\hline $\mathbf{2 0 3}$ & 0.5068 & 0.5031 & 0.5134 & 0.5065 \\
\hline $\mathbf{2 2 8}$ & 0.3274 & 0.3218 & 0.3375 & 0.3271 \\
\hline
\end{tabular}

Table.4-5 shows the performance of SSLMS, TSLMS, SSNLMS, and TSNLMS algorithms in terms of MSE and RMSE. The simulation results state that Two Stage Adaptive algorithms have minimum MSE and RMSE than single stage adaptive algorithms.

\begin{tabular}{|c|c|c|c|c|}
\multicolumn{5}{c}{ Table.6 DISTORTION for the dataset. } \\
\begin{tabular}{|c|c|c|c|} 
Record \\
No
\end{tabular} & SSLMS & TSLMS & SSNLMS & TSNLMS \\
\hline $\mathbf{1 0 0}$ & -8.5204 & -8.6364 & -8.317 & -8.5273 \\
\hline $\mathbf{1 0 5}$ & -8.0563 & -8.1566 & -7.8744 & -8.0599 \\
\hline $\mathbf{1 0 8}$ & -8.731 & -8.8488 & -8.5196 & -8.735 \\
\hline $\mathbf{2 0 3}$ & -5.904 & -5.9668 & -5.7902 & -5.9077 \\
\hline $\mathbf{2 2 8}$ & -9.6988 & -9.8484 & -9.4336 & -9.7061 \\
\hline
\end{tabular}

Table.6 shows the performance of SSLMS, TSLMS, SSNLMS and TSNLMS algorithms in terms of distortion. The simulation results state that two stage adaptive algorithms have minimum signal distortion than single stage adaptive algorithms.

\subsection{Baseline Wander (BW) Removal}

In this experiment 3600 samples of ECG signal corrupted with real baseline wander (BW of MIT-BIH NSTDB), are considered which are given as primary input to the adaptive filter of Figure 1. The Figures 5-6 depict the BW cancellation by considering the number of samples and amplitude on $\mathrm{x}$-axis and $\mathrm{y}$-axis respectively. The performance of the adaptive filter algorithms are assessed by SNR, MSE, RMSE and Distortion Values for entire data set. The two stage adaptive algorithms achieve SNR, MSE and Distortion improvement over the conventional single stage adaptive algorithms. Tables 6-8 shows improvement for the entire data set in terms of SNR, Mean Square Error (MSE) and Distortion. These results for data105 are shown in Figure 5-6.

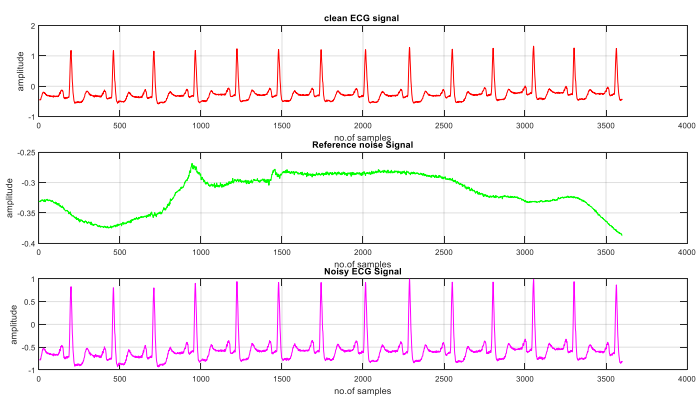

Fig. 5: Simulated model 1 filtering results of BW Cancelation (a) Clean ECG Signal(red) for MIT-BIH Record Number 105 (b) Reference BW Noise Signal (green) (c) MIT-BIH Record 105 with BW Noise (magenta)
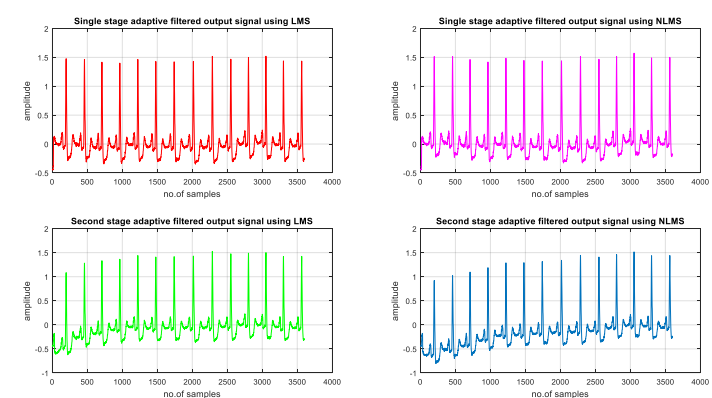

Fig. 6: Simulation results of PLI Cancelation for MIT-BIH Record Number 105 (a) Recovered (red) signal using SSLMS algorithm (b) Recovered (green) signal using TSLMS algorithm (c) Recovered(magenta) signal using SSNLMS algorithm (d) Recovered(blue) signal using TSNLMS algorithm.

\begin{tabular}{|c|c|c|c|c|c|}
\hline $\begin{array}{c}\text { Record } \\
\text { No }\end{array}$ & SNRBF & SSLMS & TSLMS & SSNLMS & TSNLMS \\
\hline 100 & 1.1285 & 1.0263 & 1.7632 & 1.1127 & 4.2437 \\
\hline 105 & 1.6261 & 3.6845 & 5.5289 & 3.1003 & 7.6927 \\
\hline 108 & 0.9082 & 1.1573 & 1.9871 & 1.2554 & 4.5957 \\
\hline 203 & 3.8812 & 8.0544 & 11.3773 & 8.0761 & 13.3679 \\
\hline 228 & -0.1391 & 1.47 & 2.7886 & 1.1666 & 5.0861 \\
\hline
\end{tabular}

Table.7 shows the performance of SSLMS, TSLMS, SSNLMS, and TSNLMS algorithms in terms of SNR..

Table.8: MSE improvement for the dataset

\begin{tabular}{|c|c|c|c|c|}
\hline $\begin{array}{c}\text { Record } \\
\text { No }\end{array}$ & SSLMS & TSLMS & SSNLMS & TSNLMS \\
\hline $\mathbf{1 0 0}$ & 0.0291 & 0.0458 & 0.0301 & 0.0956 \\
\hline $\mathbf{1 0 5}$ & 0.0906 & 0.1084 & 0.0921 & 0.1462 \\
\hline $\mathbf{1 0 8}$ & 0.025 & 0.0442 & 0.0259 & 0.0917 \\
\hline $\mathbf{2 0 3}$ & 0.2155 & 0.233 & 0.2341 & 0.2588 \\
\hline $\mathbf{2 2 8}$ & 0.0315 & 0.0489 & 0.033 & 0.0866 \\
\hline
\end{tabular}

Table.9: RMSE improvement for the dataset.

\begin{tabular}{|c|c|c|c|c|}
\hline $\begin{array}{c}\text { Record } \\
\text { No }\end{array}$ & SSLMS & TSLMS & SSNLMS & TSNLMS \\
\hline $\mathbf{1 0 0}$ & 0.1707 & 0.214 & 0.1734 & 0.3093 \\
\hline $\mathbf{1 0 5}$ & 0.301 & 0.3293 & 0.3036 & 0.3823 \\
\hline $\mathbf{1 0 8}$ & 0.1582 & 0.2103 & 0.1609 & 0.3027 \\
\hline $\mathbf{2 0 3}$ & 0.4642 & 0.4827 & 0.4838 & 0.5087 \\
\hline $\mathbf{2 2 8}$ & 0.1775 & 0.2212 & 0.1817 & 0.2943 \\
\hline
\end{tabular}

Table.8-9 shows the performance of SSLMS, TSLMS, SSNLMS, and TSNLMS algorithms in terms of MSE and RMSE. The simulation results state that Two Stage Adaptive algorithms have minimum MSE and RMSE than single stage adaptive algorithms.

Table.10: DISTORTION for the dataset.
\begin{tabular}{|c|c|c|c|c|}
\hline $\begin{array}{c}\text { Record } \\
\text { No }\end{array}$ & SSLMS & TSLMS & SSNLMS & TSNLMS \\
\hline $\mathbf{1 0 0}$ & -15.3547 & -13.3927 & -15.2184 & -10.1936 \\
\hline $\mathbf{1 0 5}$ & -10.4297 & -9.6481 & -10.3551 & -8.3516 \\
\hline $\mathbf{1 0 8}$ & -16.0143 & -13.5417 & -15.8662 & -10.3784 \\
\hline $\mathbf{2 0 3}$ & -6.6654 & -6.3263 & -6.3068 & -5.8704 \\
\hline $\mathbf{2 2 8}$ & -15.0166 & -13.1058 & -14.8107 & -10.6234 \\
\hline
\end{tabular}

Table.10 shows the performance of SSLMS, TSLMS, SSNLMS and TSNLMS algorithms in terms of distortion. The simulation results state that two stage adaptive algorithms have minimum signal distortion than single stage adaptive algorithms.

\section{Conclusions}

This paper presents the adaptive noise cancellation of ECG Signals using two stage adaptive filters. The algorithm is same as the traditional methods using gradient descent technique with single adaptive filter. However, the proposed model can potentially be a new implementation form of ANC algorithm. Two stage adaptive algorithm guarantees a more stable conversion in response to variations in input signal power. The mathematical analysis of two 
stage ANC algorithm is carried out, and its simulation is performed successfully using MATLAB 2013a. In order to study the performance of system, a comparison has been made between single and Two stage ANC. The proposed two stages ANC consist of noise and clean ECG signal as input parameters for which adaptive filtered output signal, error signal and filter weights are obtained as output parameters. The performance of single stage ANC and two stage ANC are compared on the basis of output signal, error signal, and filter weights, SNR, MSE, RMSE and Distortion. The two stage adaptive filter is much efficient in terms of noise cancellation than single stage adaptive filter. Simulation results demonstrate that the proposed method achieves good adaptation and performance in biomedical signal processing field. The signalto-noise ratio for various filters of two stages ANC was found to be higher than Single Stage ANC system.

\section{References}

[1] Catalano, Joseph T. Guide to ECG analysis. Lippincott Williams Wilkins, 2002.

[2] Katz, Arnold M. Physiology of the Heart. Lippincott Williams Wilkins, 2010.

[3] Manolakis, Dimitris G., Vinay K. Ingle, and Stephen M. Kogon Statistical and adaptive signal processing: spectral estimation, signal modeling, adaptive filtering, and array processing. Boston: McGraw-Hill, 2000.

[4] Diniz, P. S. R. "Adaptive Filtering: Algorithms and Practical Implementation. Springer." New York, NY, USA (2008).

[5] Haykin, Simon S. Adaptive filter theory. Pearson Education India, 2008.

[6] Kuo, Sen M., and Dennis Morgan. Active noise control systems algorithms and DSP implementations. John Wiley Sons, Inc. 1995

[7] John G.. Proakis, and Dimitris G.. Manolakis. Digital signal processing: principles, algorithms, and applications. Pearson Prentice Hall, 2007.

[8] Widrow, Bernard, et al. "Adaptive noise cancelling: Principles and applications." Proceedings of the IEEE 63.12 (1975): 1692 1716

[9] Hayes, Monson H. Statistical digital signal processing and modeling. John Wiley Sons, 2009.

[10] Mandic, Danilo P. "A generalized normalized gradient descent algorithm." IEEE Signal Processing Letters 11.2 (2004): 115 118.

111] Van Alste, Jan A., and T. S. Schilder. "Removal of base-line wander and power-line interference from the ECG by an efficient FIR filter with a reduced number of taps." IEEE transactions on biomedical engineering 12 (1985): 1052-1060.

[12] Chavan, Mahesh S., R. A. Agarwala, and M. D. Uplane. "Suppression of Baseline Wander and power line interference in ECG using Digital IIR Filter.” InternationaL journal of circuits, systems and signal processing 2.2 (2008): 356-365.

[13] Shantha Selva, S. Bharathi, and V. Sadasivam. "Design of Optimal Discrete Wavelet for ECG Signal using orthogonal filter bank." Conference on Computational Intelligence and Multimedia Applications, 2007. International Conference on. Vol. 1. IEEE, 2007.

[14] Hargittai, S. "Savitzky-Golay least-squares polynomial filters in ECG signal processing." Computers in Cardiology, 2005. IEEE, 2005.

[15] Weiting, Yuan, and Zhou Runjing. "An improved self-adaptive filter based on LMS algorithm for filtering $50 \mathrm{~Hz}$ interference in ECG signals." Electronic Measurement and Instruments, 2007. ICEMI'07. 8th International Conference on. IEEE, 2007.

[16] Rahman, Mohammad Zia Ur, Rafi Ahamed Shaik, and DV Rama Koti Reddy. "Adaptive noise removal in the ECG using the block LMS algorithm." Adaptive Science Technology, 2009 ICAST 2009. 2nd International Conference on. IEEE, 2009.

[17] Sankar, A. Bhavani, D. Kumar, and K. Seethalakshmi. "Performance study of various adaptive filter algorithms for noise cancellation in respiratory signals." Signal processing: An international journal (SPIJ) 4.5 (2010): 267

[18] Rahman, Mohammad Zia Ur, Rafi Ahamed Shaik, and DV Rama Koti Reddy. "Noise cancellation in ECG signals using normalized SignSign LMS algorithm." Signal Processing and Information Technology (ISSPIT), 2009 IEEE International Symposium on. IEEE, 2009.
[19] Dixit, Shubhra, and Deepak Nagaria. "Design and analysis of cascaded lms adaptive filters for noise cancellation." Circuits, Systems, and Signal Processing 36.2 (2017): 742-766.

[20] Sun, Xu, and Sen M. Kuo. "Active narrowband noise control systems using cascading adaptive filters." IEEE Transactions on Audio, Speech, and Language Processing 15.2 (2007): 586-592

[21] Sharma, Arpit, Sandeep Toshniwal, and Richa Sharma. "Noise reduction technique for ECG signals using adaptive filters." Int J Res Rev 7.2 (2014): 187-191.

[22] Rahman, Md Zia Ur, Rafi Ahamed Shaik, and D. V. R. K. Reddy. "Noise cancellation in ECG signals using computationally simplified adaptive filtering techniques: Application to biotelemetry." Signal Processing: An International Journal (SPIJ) 3.5 (2009): 1-12.

[23] Abadi, Mohammad Shams Esfand, and Ali Mahlooji Far. "A unified framework for adaptive filter algorithms with variable step-size." Computers Electrical Engineering 34.3 (2008): 232 249

[24] Huang, Hsu-Chang, and Junghsi Lee. "A new variable step-size NLMS algorithm and its performance analysis." IEEE Transactions on Signal Processing 60.4 (2012): 2055-2060.

[25] Gorriz, Juan Manuel, et al. "A novel LMS algorithm applied to adaptive ' noise cancellation." IEEE Signal Processing Letters16.1 (2009): 34-37.

[26] Huang, Hsu-Chang, and Junghsi Lee. "A new variable step-size NLMS algorithm and its performance analysis." IEEE Transactions on Signal Processing 60.4 (2012): 2055-2060.

[27] Sankar, A. Bhavani, D. Kumar, and K. Seethalakshmi. "Performance study of various adaptive filter algorithms for noise cancellation in respiratory signals." Signal processing: An international journal (SPIJ) 4.5 (2010): 267.

[28] Sameni, Reza, M. B. Shamsollahi, and Christian Jutten. "Filtering electrocardiogram signals using the extended Kalman filter.' Engineering in Medicine and Biology Society, 2005. IEEEEMBS 2005. 27th Annual International Conference of the. IEEE 2005.

[29] Yazdanpanah, Babak, K. Sravan Kumar, and G. S. N Raju. "Noise removal ecg signal using non-adaptive filters and adaptive filter algorithm." 2015 International Conference on Electrical, Electronics, Signals, Communication and Optimization (EESCO). 2015.

[30] Popescu, M., P. Cristea, and A. Bezerianos. "High resolution ECG filtering using adaptive Bayesian wavelet shrinkage." Computers in Cardiology 1998. IEEE, 1998.

[31] Barros, Allan Kardec, Ali Mansour, and Noboru Ohnishi. "Removing artifacts from electrocardiographic signals using independent components analysis." Neurocomputing 22.1 (1998): 173-186.

[32] He, Taigang, Gari Clifford, and Lionel Tarassenko. "Application of independent component analysis in removing artefacts from the electrocardiogram." Neural Computing Applications 15.2 (2006): 105-116.

[33] Jenitta, J., and A. Rajeswari. "Denoising of ECG signal based on improved adaptive filter with EMD and EEMD." Information Communication Technologies (ICT), 2013 IEEE Conference on. IEEE, 2013.

[34] Chang, Kang-Ming. "Arrhythmia ECG noise reduction by ensemble empirical mode decomposition." Sensors 10.6 (2010): 6063-6080.

[35] Jenitta, J., and A. Rajeswari. "Denoising of ECG signal based on improved adaptive filter with EMD and EEMD." Information Communication Technologies (ICT), 2013 IEEE Conference on. IEEE, 2013.

[36] Donoho, David L. "De-noising by soft-thresholding." IEEE transactions on information theory 41.3 (1995): 613-627.

[37] Agante, P. M., and JP Marques De Sa. "ECG noise filtering using ' wavelets with soft-thresholding methods." Computers in Cardiology, 1999. IEEE, 1999.

[38] Sayadi, Omid, and Mohammad B. Shamsollahi. "Multiadaptive bionic wavelet transform: Application to ECG denoising and baseline wandering reduction." EURASIP Journal on Advances in Signal Processing 2007.1 (2007): 041274.

[39] Singh, Brij N., and Arvind K. Tiwari. "Optimal selection of wavelet basis function applied to ECG signal denoising." Digital signal processing 16.3 (2006): 275-287.

[40] Moody, George B., and Roger G. Mark. "QRS morphology representation and noise estimation using the Karhunen-Loeve transform." Computers in Cardiology 1989, Proceedings.. IEEE, 1989. 
[41] Mahmoodabadi, S. Z., et al. "ECG feature extraction based on multiresolution wavelet transform." Engineering in Medicine and Biology Society, 2005. IEEE-EMBS 2005. 27th Annual International Conference of the. IEEE, 2006.

[42] Daqrouq, K. "ECG baseline wandering reduction using discrete wavelet transform." Asian Journal of Information Technology 4.11 (2005): 989- 995.

[43] Kestler, H. A., et al. "De-noising of high-resolution ECG signals by combining the discrete wavelet transform with the Wiener filter." Computers in Cardiology 1998. IEEE, 1998.

[44] Poungponsri, Suranai, and Xiao-Hua Yu. "An adaptive filtering approach for electrocardiogram (ECG) signal noise reduction using neural networks." Neurocomputing 117 (2013): 206-213.

[45] Jagtap, S. K., et al. "Application of the digital filter for noise reduction in electrocardiogram." Journal of Instrumentation40.2 (2010): 83-86.

[46] Singh, Sachin, and K. L. Yadav. "Performance evaluation of different adaptive filters for ECG signal processing." International Journal On Computer Science and Engineering2.5 (1880): 2010.

[47] Kærgaard, Kevin, Søren Hjøllund Jensen, and Sadasivan Puthusserypady. "A comprehensive performance analysis of EEMD-BLMS and DWT-NN hybrid algorithms for ECG denoising.” Biomedical Signal Processing and Control 25 (2016): 178-187.

[48] Mark, R., and G. Moody. "MIT-BIH arrhythmia database directory." Cambridge: Massachusetts Institute of Technology(1988).

[49] Reddy, D. C. Biomedical signal processing: principles and techniques. McGraw-Hill, 2005.

[50] Joshi, Sarang L., Rambabu A. Vatti, and Rupali V. Tornekar. "A survey on ECG signal denoising techniques." Communication Systems and Network Technologies (CSNT), 2013 International Conference on. IEEE, 2013.

[51] Almenar, Vincenc, and Antonio Albiol. "A new adaptive scheme for ECG enhancement.” Signal Processing 75.3 (1999): 253-263.

[52] Islam, Syed Zahurul, et al. "Performance study of adaptive filtering algorithms for noise cancellation of ECG signal." Information, Communications and Signal Processing, 2009. ICICS 2009. 7th International Conference on. IEEE, 2009. [53] Goldberger, A. L., et al. "The MIT-BIH normal sinus rhythm database." Online], 